\title{
ARTICLE OPEN \\ First-principles treatment of Mott insulators: linearized QSGW+DMFT approach
}

\author{
Sangkook Choi ${ }^{1,2}$, Andrey Kutepov ${ }^{2}$, Kristjan Haule ${ }^{2}$, Mark van Schilfgaarde ${ }^{3}$ and Gabriel Kotliar ${ }^{1,2}$
}

The theoretical understanding of emergent phenomena in quantum materials is one of the greatest challenges in condensed matter physics. In contrast to simple materials such as noble metals and semiconductors, macroscopic properties of quantum materials cannot be predicted by the properties of individual electrons. One of the examples of scientific importance is strongly correlated electron system. Neither localized nor itinerant behaviors of electrons in partially filled $3 d, 4 f$, and $5 f$ orbitals give rise to rich physics such as Mott insulators, high-temperature superconductors, and superior thermoelectricity, but hinder quantitative understanding of low-lying excitation spectrum. Here we present a new first-principles approach to strongly correlated solids. It is based on a combination of the quasiparticle self-consistent GW approximation and the dynamical mean-field theory. The sole input in this method is the projector to the set of correlated orbitals for which all local Feynman graphs are being evaluated. For that purpose, we choose very localized quasiatomic orbitals spanning large energy window, which contains most strongly hybridized bands, as well as upper and lower Hubbard bands. The self-consistency is carried out on the Matsubara axis. This method enables the first-principles study of Mott insulators in both their paramagnetic and antiferromagnetic phases. We illustrate the method on the archetypical charge transfer correlated insulators $\mathrm{La}_{2} \mathrm{CuO}_{4}$ and $\mathrm{NiO}$, and obtain spectral properties and magnetic moments in good agreement with experiments.

npj Quantum Materials (2016) 1, 16001; doi:10.1038/npjquantmats.2016.1; published online 27 July 2016

\section{INTRODUCTION}

The first-principles description of strongly correlated materials is currently regarded as one of the greatest challenges in condensed matter physics. The interplay between correlated electrons in open $d$ - or $f$ - shell and itinerant band states gives rise to rich physics that makes these materials attractive for a wide range of applications such as oxide electronics, high-temperature superconductors, and spintronic devices. Various theoretical approaches are currently being pursued. ${ }^{1}$ One of the most successful approaches is the dynamical mean-field theory (DMFT). $^{2}$ In combination with density functional theory, ${ }^{3,4}$ it has described many features of strongly correlated materials successfully and highlighted the surprising accuracy of treating correlations local to a small subset of orbitals exactly, while treating the remainder of the problem in a static mean-field manner. ${ }^{5}$

The numerous successes of DMFT in different classes of correlated materials revived the interest in the long-sought goal of achieving a diagrammatically controlled approach to the quantum many-body problem of solids, starting from the Green's function $G$ and the screened Coulomb interactions $W^{6,7}$ The lowest order diagram in perturbation theory in this functional gives rise to the GW approximation, ${ }^{8}$ whereas the local approximation applied to the most correlated orbitals gives rise to an extended DMFT approach to the electronic structure problem. ${ }^{7}$ The addition of the GW and DMFT graphs was proposed and implemented in model Hamiltonian studies ${ }^{9}$ and in realistic electronic structure. ${ }^{10,11}$ There is now intense activity in this area with many recent publications ${ }^{12-15}$ triggered by advances in the quality of the impurity solvers, ${ }^{16,17}$ insights into the analytic form of the high-frequency behavior of the self-energy, ${ }^{18}$ and improved electronic structure codes.

Several conceptual issues remain to be clarified before the long-sought goal of a robust electronic structure method for solids is attained. The first issue is the choice of local orbitals on which to perform the DMFT method (summation of all local Feynman graphs). The second issue is the level of self-consistency that should be used in the calculation of various parts of the diagrams included in the treatment (free or bare Green's function $G_{0}$ versus self-consistent interacting Green's functions $G$ ). These central issues are addressed in this letter.

The self-consistency issue appears already at the lowest order, namely the GW level and it has been debated over time. The corresponding issue in GW+DMFT is expected to be at least as important, but has not been explored, except for model Hamiltonians. ${ }^{19,20}$ At the GW level, it is now well established that Hedin's fully self-consistent formulation, ${ }^{8}$ while producing good total energies in solids, ${ }^{21}$ atoms, and molecules, ${ }^{22,23}$ does not produce a good approximation to the spectra of even threedimensional electron gas and aluminum in comparison with nonself-consistent GW results. ${ }^{21,24}$ Instead, using a free (quasiparticle (QP)) Green's function in the evaluation of the polarization graph of the GW method gives much better results for spectral functions. This is the basis of the one-shot QP GW, starting from local density approximation (LDA) ${ }^{25}$ or from others. ${ }^{26}$ Unfortunately, the answer depends on the starting point. A solution for this problem is to impose a self-consistency equation to determine $G_{0}$. This method, called the QP self-consistent GW (QSGW), ${ }^{27}$ is very successful reproducing the spectra of many systems. ${ }^{27}$ How to combine it with DMFT is an important open challenge. ${ }^{28,29}$

${ }^{1}$ Condensed Matter Physics and Materials Science Department, Brookhaven National Laboratory, Upton, NY, USA; ${ }^{2}$ Department of Physics and Astronomy, Rutgers University, Piscataway, NJ, USA and ${ }^{3}$ Department of Physics, Kings College London, Strand, London, UK.

Correspondence: G Kotliar (kotliar@physics.rutgers.edu)

Received 11 April 2016; revised 4 May 2016; accepted 5 May 2016 
Previous GW+DMFT studies typically used a $G_{0}$, which depends on the LDA starting point, and projectors spanning a relatively small energy window. ${ }^{12-15}$ In this work, we propose a different approach to the level of self-consistency and the choice of the DMFT orbital. We do a self-consistent QSGW calculation and then calculate local self-energy using DMFT with static $U_{\mathrm{d}}$ and $J_{\mathrm{H}}$ without feedback to non-local self-energy within GW. For the DMFT step, we choose a very localized orbital spanning large energy window, which contains most strongly hybridized bands, as well as upper and lower Hubbard bands.

In the LDA+DMFT context, the choice of very localized orbitals has provided a great deal of universality, as the interactions do not vary much among compounds of the same family. This has been demonstrated in the studies of iron pnictides ${ }^{30}$ and transition metal oxides. ${ }^{31}$ This choice results in a second advantage as we will show below, namely the frequency dependence of the interaction matrix can be safely ignored. Having chosen the correlated orbitals, all the other parameters are self-consistently determined. This is the first $a b$ initio QP self-consistent GW+DMFT implementation and the first study on a paramagnetic (PM) Mott insulator within the GW+DMFT method.

\section{RESULTS}

Figure $2 \mathrm{a}$ shows the frequency dependence of real and imaginary parts of $U_{\mathrm{d}}$ of $\mathrm{La}_{2} \mathrm{CuO}_{4}$ shown in Figure 1. It is calculated on an imaginary frequency axis and analytically continued by a maximum entropy method. ${ }^{32}$ We also plot the fully screened Coulomb interaction $W_{\mathrm{d}}$ for comparison. Static $U_{\mathrm{d}}$ is $12.0 \mathrm{eV}$ and $U_{\mathrm{d}}$ remains almost constant up to $10 \mathrm{eV}$. In contrast, in $W_{\mathrm{d}}$, there are several peaks due to low-energy collective excitations below $10 \mathrm{eV}$. At very high energy, $U_{d}$ approaches the bare coulomb interaction of $28 \mathrm{eV}$. Static value of $U_{\mathrm{pd}}$ is $2.0 \mathrm{eV}$, much smaller than $U_{\mathrm{d}}$; hence, we do not discuss its treatment further (The renormalization of $U_{\mathrm{d}}$ by the nonlocal Coulomb interaction can be understood by using the definition of Hubbard $U$ by $C$ Herring. According to Herring, ${ }^{33}$ the Hubbard $U$ can be obtained from the reaction of $2\left(d^{n_{d}} p^{n_{p}}\right) \rightarrow d^{n_{d}+1} p^{n_{p}-1}+d^{n_{d}-1} p^{n_{p}+1}$ between Cu- $d$ and O-p orbitals, where $n_{\mathrm{d}}$ and $n_{\mathrm{p}}$ are the number of electrons in $\mathrm{Cu}-d$ and $\mathrm{O}-p$ orbitals, respectively. The energy cost

a



Figure 1. Atomic structure and first Brillouin zone of $\mathrm{La}_{2} \mathrm{CuO}_{4}$. (a) Atomic structure of $\mathrm{La}_{2} \mathrm{CuO}_{4}$ in the single face-centered orthorhombic phase. Lanthanum atoms are represented by green spheres, copper atoms by blue spheres in the blue octahedrons, and oxygen atoms by red spheres. The structure is characterized by an alternating rotation of successive $\mathrm{CuO}_{6}$ octahedra along the $x$ direction. (b) First Brillouin zone of single face-centered orthorhombic phase. Red lines show the path along which electronic band structures are plotted in Figures $2 c$ and 3. for the reaction is approximately $U_{d}-2 U_{p d}+U_{p}$ and this should be the sum of the renormalized $U_{\mathrm{d}}$ and renormalized $U_{\mathrm{p}}$. In the simplest case, where we have one electron in $p$ and $d$ orbitals, renormalized $\mathrm{U}$ of $\mathrm{Cu}-d$ orbitals is $U_{\mathrm{d}}-U_{\mathrm{pd}}$ from the reaction of $d^{1} p^{1} \rightarrow d^{2} p^{0}$. The renormalized $U_{\mathrm{d}}$ is derived more rigorously by Schüler et $a l^{34}$ ). Calculated $J_{\mathrm{H}}$ is $1.4 \mathrm{eV}$ and has negligible frequency dependence. By contrast, conventional constrained random phase approximation, in which 10 bands of mostly $\mathrm{Cu}-3 d$ character are excluded from screening, results in static $U_{d}=7.6 \mathrm{eV}$, which is too small to open the Mott gap, and which is also inconsistent with photoemission experiments on $\mathrm{CuO}$ charge transfer insulators. ${ }^{35}$

We introduced a complementary method to compute the static $U_{\mathrm{d}}$. The key idea is to first calculate the excitation spectra of $\mathrm{La}_{2} \mathrm{CuO}_{4}$ within Matsubara QSGW (MQSGW)+DMFT using local GW (with a static $U_{\mathrm{d}}$ ) as the impurity solver and then determine $U_{\mathrm{d}}$, by finding the value that best matches the full spin-polarized MQSGW spectra. The procedure starts from the non-spinpolarized MQSGW band structure without magnetic long-range order. We then allow spontaneous magnetic long-range order by embedding a polarized impurity self-energy for the $\mathrm{Cu}-3 d$ electrons computed in a local GW approximation. We find that indeed magnetic ordering associated with $\mathrm{Cu}-3 d$ is captured by spin-polarized local MQSGW using a static value of $U_{\mathrm{d}}$ and $J_{\mathrm{H}}$, and spectral properties such as energy gap are very similar in value to the full spin-polarized MQSGW spectra. In Figure 2b, we allowed $U_{\mathrm{d}}$ to vary between 8 and $13 \mathrm{eV}$ (at fixed $J_{\mathrm{H}}=1.4 \mathrm{eV}$ ) and we plot the size of the indirect gap. The gap size of this method matches


Figure 2. Hubbard $\mathrm{U}$ associated with $\mathrm{Cu}-3 d$ orbitals in $\mathrm{La}_{2} \mathrm{CuO}_{4}$. (a) Frequency dependence of $W_{\mathrm{d}}$ (dashed lines) and $U_{\mathrm{d}}$ (full lines) of $\mathrm{La}_{2} \mathrm{CuO}_{4}$ with a $X_{Q P}^{\text {low }}$ defined in the energy window $E_{\mathrm{F}} \pm 10 \mathrm{eV}$. Real and imaginary parts of the parameter are marked by red and blue colors, respectively. (b) Bandgap dependence on $U_{d}$, in $\mathrm{La}_{2} \mathrm{CuO}_{4}$, evaluated with impurity self-energy within spin-polarized GW approximation with $J_{H}=1.4 \mathrm{eV}$. The black dashed line represents bandgap within spin-polarized Matsubara QP self-consistent GW (MQSGW). (c) Spectral function of $\mathrm{La}_{2} \mathrm{CuO}_{4}$ with $U_{\mathrm{d}}=12 \mathrm{eV}$ and $J_{H}=1.4 \mathrm{eV}$. The black dashed-lines show band structures within spinpolarized MQSGW. 
the gap of spin-polarized MQSGW when $U_{\mathrm{d}} \approx 12 \mathrm{eV}$. If this choice of $U_{\mathrm{d}}$ and $J_{\mathrm{H}}$ is correct, the resulting spectra must be similar to the prediction of spin-polarized MQSGW method. We show this comparison in Figure $2 c$ to confirm a good match. In addition, the relative position of the $\mathrm{Cu}-d$ band (the lowest energy conduction band at S) to the La- $d$ band (the lowest energy conduction band at $Y)$ is also well matched, justifying the approximation of $\hat{\Sigma}^{D C}\left(i \omega_{n}\right) \simeq \hat{\Sigma}^{D C}\left(i \omega_{n}=0\right) . \Sigma^{D C}\left(i \omega_{n}=0\right)$ for $C u-d_{x^{2}}-y^{2}$ orbital differs from nominal double counting energy ${ }^{36}$ by only $1 \%$, highlighting again the advantages of using a broad window and narrow orbitals.

We now discuss the magnetic moment associated with $\mathrm{Cu}$ and the electronic excitation spectra of $\mathrm{La}_{2} \mathrm{CuO}_{4}$ by using MQSGW +DMFT (with $U_{\mathrm{d}}=12.0 \mathrm{eV}, J_{\mathrm{H}}=1.4 \mathrm{eV}$ ) in which the impurity is solved by the numerically exact continuous-time quantum MonteCarlo ${ }^{16,17}$ and compare them with other methods. Local spin density approximation does not have a magnetic solution. In contrast, spin-polarized MQSGW, QSGW, ${ }^{27}$ and MQSGW+DMFT predict $0.7 \mu_{\mathrm{B}}, 0.7 \mu_{\mathrm{B}}$, and $0.8 \mu_{\mathrm{B}}$, respectively. This is consistent with experimental measurements, although the later span quite large range $0.4 \sim 0.8 \mu_{\mathrm{B}}{ }^{37-39}$

In the low-energy spectrum of $\mathrm{La}_{2} \mathrm{CuO}_{4}$, local spin density approximation does not have an insulating solution; there is a single non-magnetic solution with zero energy gap as shown in the bandstructure(Figure 3a) and total density of states (Figure 4a). The non-spin-polarized MQSGW also predicts metal as shown in Figure $4 a$, but the two bands of primarily $\mathrm{Cu}-d_{x^{2}}-y^{2}$ character near the Fermi level are well-separated from the rest of the bands (dashed lines in Figure 3b). Spin-polarized MQSGW calculation (dashed lines in Figure $3 c$ ) yields qualitative different results from local spin density approximation and non-spinpolarized MQSGW calculation. The two $\mathrm{Cu}-d_{x^{2}-y^{2}}$ bands are now well separated from each other with a bandgap of $3.4 \mathrm{eV}$. Spin-polarized QSGW ${ }^{27}$ also yields insulating phase with a gap of $4.0 \mathrm{eV}$. In the experiment, the larger direct gap, as measured by optics, is $\sim 2 \mathrm{eV} .40,41$

We show that these deficiencies of LDA, QSGW and MQSGW in the low-energy spectra can be remedied by adding all local Feynman diagrams for the $\mathrm{Cu}-d$ orbitals using the DMFT. The LDA +DMFT calculation in Figure 4a, carried out by the all-electron LDA +DMFT method, ${ }^{31,36}$ predicts reasonable gap of 1.5 and $1.8 \mathrm{eV}$ in PM and antiferromagnetic (AFM) phases, in good agreement with experiment and previous LDA+DMFT studies. ${ }^{31,42-45}$ Within MQSGW+DMFT, we find gaps of 1.5 and $1.6 \mathrm{eV}$ in PM and AFM phases, respectively, as shown in Figure $4 b$. The excitation spectra of MQSGW+DMFT in PM and AFM phase as shown in Figure $3 b, c$ are very similar, as both are insulating with well-separated

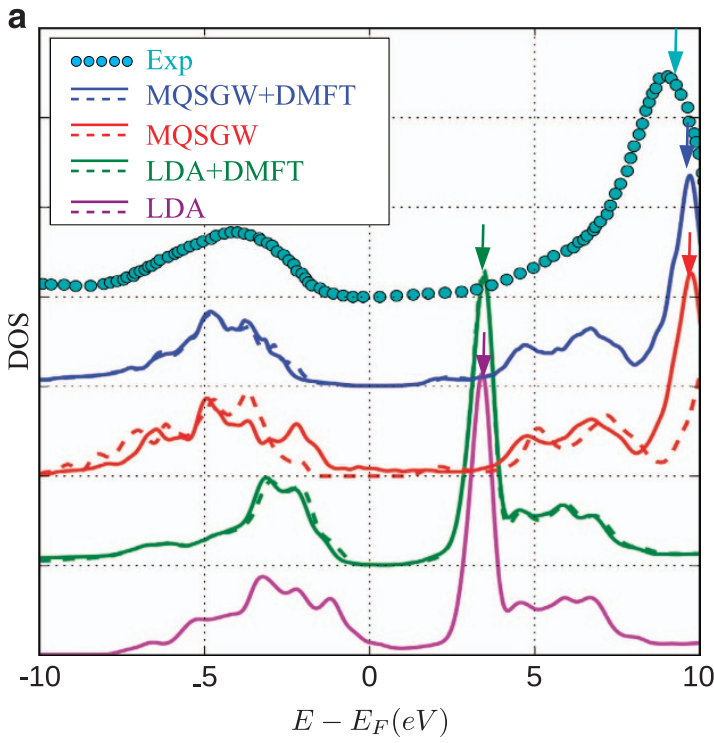

b
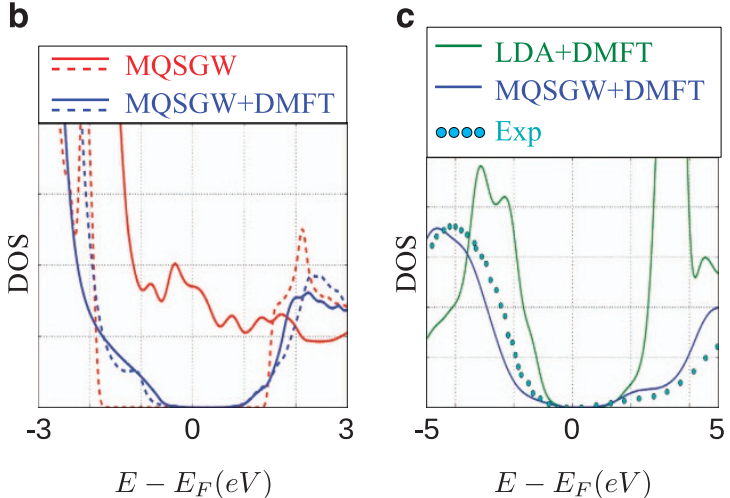

Figure 4. The density of states of $\mathrm{La}_{2} \mathrm{CuO}_{4}$. (a) Total density of states of $\mathrm{La}_{2} \mathrm{CuO}_{4}$ from local density approximation (LDA) (magenta), LDA +dynamical mean-field theory (DMFT) (green), Matsubara QP selfconsistent GW (MQSGW) (red), and MQSGW+DMFT (blue). Full lines and dashed lines represent quantities within non-spin-polarized and spin-polarized versions of each calculation, respectively. The cyan dotted line shows photoemission/inverse photoemission data. ${ }^{46}$ The positions of La-f peaks are marked by arrows. (b) A zoom-in view in the low-energy region. (c) The overlap of total density of states of $\mathrm{La}_{2} \mathrm{CuO}_{4}$ within LDA+DMFT as well as MQSGW+DMFT and photoemission/inverse photoemission data. ${ }^{46}$
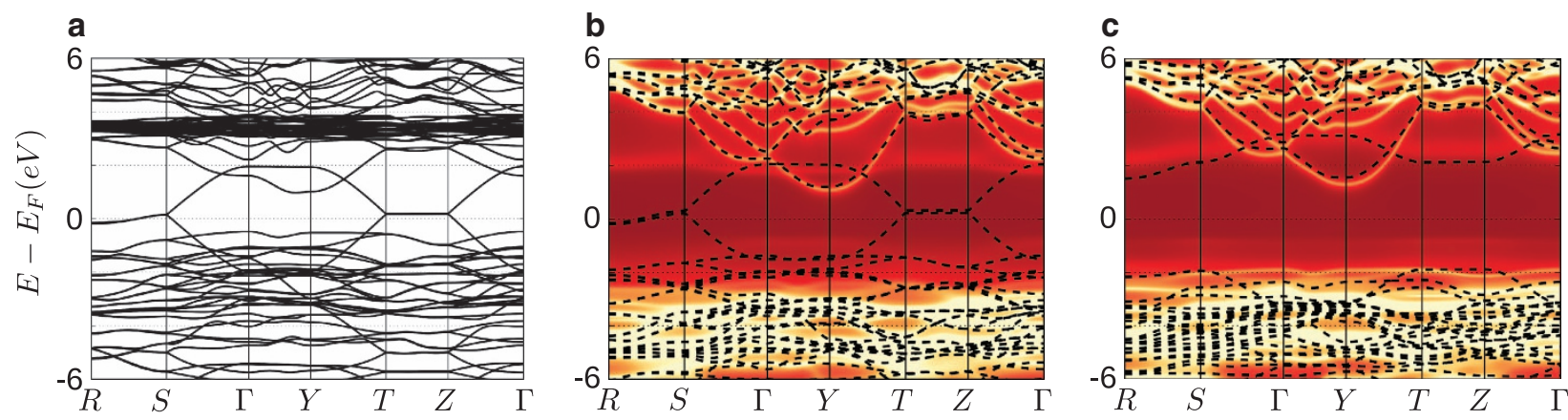

Figure 3. The low-energy spectral function of $\mathrm{La}_{2} \mathrm{CuO}_{4}$. (a) Electronic bandstructures of $\mathrm{La}_{2} \mathrm{CuO}_{4}$ within local spin density approximation and spectral functions from (b) non-spin-polarized Matsubara QP self-consistent GW (MQSGW)+dynamical mean-field theory (DMFT) (c) and spinpolarized MQSGW+DMFT calculations along the path shown in Figure 1b. The dashed lines in $\mathbf{b}, \mathbf{c}$ represent electronic band structures within non-spin-polarized MQSGW and spin-polarized MQSGW, respectively. 
Cu- $d_{x^{2}-y^{2}}$ bands, which is now also substantially broadened due to large scattering rate in Hubbard-like bands. In addition, MQSGW +DMFT improves the line-shape of LDA+DMFT. Near the top of the valence bands with oxygen $p$ character, the lineshape within LDA + DMFT is too sharp in comparison with the experiments as shown in Figure 4c. By treating oxygen $p$ levels within GW, the lineshape becomes smoother and in a better agreement with experiments.

In the high-energy region of $\mathrm{La}_{2} \mathrm{CuO}_{4}$, the most distinctive difference is the position of La-f peak. It appears at $\sim 3 \mathrm{eV}$ within LDA and LDA+DMFT, but at around $\sim 9 \mathrm{eV}$ in the inverse-photoemission spectra (cyan dotted line in Figure $4 a$ ). ${ }^{46}$ By treating La- $f$ within GW approximation, it appears at $\sim 10 \mathrm{eV}$ within MQSGW and MQSGW+DMFT. The underestimation of unoccupied La-f excitation energy is attributed to the local approximation to the electron self-energy within LDA. Within LDA, Hartree and exchange-correlation potential applied to La-f orbitals are orbital independent, as charge density is averaged over 14 different $\mathrm{m}$ channels. ${ }^{47}$ In contrast, these potentials within MQSGW are orbital dependent and non-local. The effect of orbital-dependent potential can be tested within LDA+U approaches, as LDA $+U$ adds orbital-dependent potential and subtracts orbital-independent potential explicitly ${ }^{3}$. From LDA+U approaches, we can also understand MQSGW better, as LDA+U can be regarded as a local and static approximation to GW approximation $^{3}$. According to Czyzyk and Sawatzky ${ }^{48}$, La-f peaks shift from $E_{\mathrm{F}}+3 \mathrm{eV}$ to $E_{\mathrm{F}}+3 \mathrm{eV}+U / 2$ with $U=11 \mathrm{eV}$ for La-f.

We also tested our proposed scheme with one more charge transfer insulator, NiO. Figure 5 a shows the frequency dependence of $U_{d}$ and $W_{d}$ for the Ni-3d orbitals in the low-energy region. In contrast to $W_{\mathrm{d}}, U_{\mathrm{d}}$ is almost constant up to $5 \mathrm{eV}$. Static $U_{\mathrm{d}}$ is $9.6 \mathrm{eV}$. In the high-energy limit, $U_{d}$ and $W_{d}$ approach the bare value of

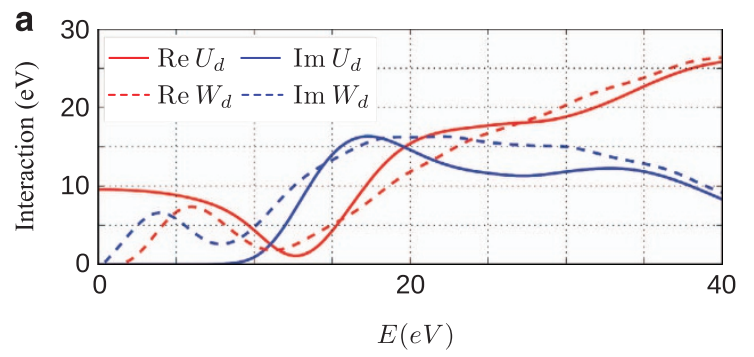

b

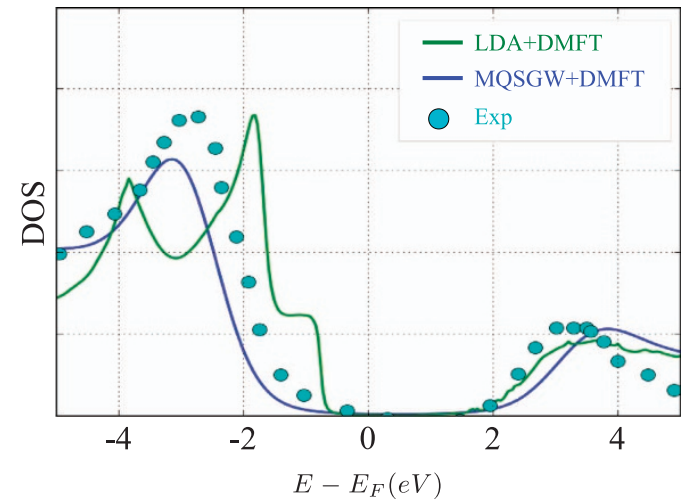

Figure 5. Hubbard $U$ associated with $\mathrm{Ni}-3 d$ orbitals in $\mathrm{NiO}$ (a) Frequency dependence of $W_{d}$ (dashed lines) and $U_{d}$ (full lines) of $\mathrm{NiO}$, with a $X_{\mathrm{QP}}^{\text {low }}$ defined in the energy window in $E_{\mathrm{F}}-11 \mathrm{eV}$ to $E_{\mathrm{F}}+10 \mathrm{eV}$. Real and imaginary parts of the parameter are marked by red and blue colors, respectively. (b) Total density of states of $\mathrm{NiO}$ within LDA+dynamical mean-field theory (DMFT) (green) and Matsubara QP self-consistent GW (MQSGW)+DMFT (blue). The cyan dotted line shows photoemission/inverse photoemission data. ${ }^{49}$
$26.0 \mathrm{eV}$. Calculated $J_{\mathrm{H}}$ for the $\mathrm{Ni}-3 d$ orbitals has negligible frequency dependence and static $J_{H}$ is $1.4 \mathrm{eV}$. Figure $5 \mathrm{~b}$ shows the total density of states of $\mathrm{NiO}$ within LDA+DMFT and MQSGW + DMFT in its PM phase. Photoemission/inverse photoemission data are also plotted for comparison. ${ }^{49}$ The LDA+DMFT calculation is being carried out by the all-electron LDA+DMFT method ${ }^{36}$ with $U_{\mathrm{d}}=10 \mathrm{eV}, J_{\mathrm{H}}=0.9 \mathrm{eV}$ and nominal double-counting energy. In the PM phase, LDA+DMFT and MQSGW+DMFT predict insulator in an agreement with previous LDA+DMFT studies, ${ }^{50,51}$ but MQSGW +DMFT improves the lineshape of LDA+DMFT. Near the top of the valence bands, the lineshape within LDA+DMFT is too sharp in comparison with the experiments. By treating oxygen $p$ levels within GW, the lineshape becomes smoother and in a better agreement with experiments. In the AFM phase, magnetic moment associated with $\mathrm{Ni}-d$ orbitals is $1.6 \mu_{\mathrm{B}}$ within MQSGW +DMFT, in agreement with experimental value of $1.6-1.9 \mu_{\mathrm{B}}{ }^{50,52,53}$

In summary, we introduced a new methodology within MQSGW +DMFT and tested it in the classic charge transfer insulator $\mathrm{La}_{2} \mathrm{CuO}_{4}$ and $\mathrm{NiO}$. Our methodology predicts a Mott-insulating gap in the PM phase, thus overcoming the limitation of LDA and QSGW. It yields more precise peak positions of the La-f states in $\mathrm{La}_{2} \mathrm{CuO}_{4}$ and valence band lineshape, thus improving the results of LDA+DMFT. The method should be useful in understanding electronic excitation spectrum of other strongly correlated materials, in particular, those where precise position of both the itinerant and correlated states is important.

\section{METHODS}

Our approach is carried out entirely on the Matsubara axis, which requires a different approach to the $\mathrm{QP}$ self-consistency in $\mathrm{GW}^{54}$ called MQSGW, where the QP Hamiltonian is constructed by linearizing the self-energy and renormalization factor (MQSGW is a form of QP self-consistency that replaces $\hat{\Sigma}\left(i \omega_{n}\right)$ with $\hat{\Sigma}(0)+i \omega_{n} \hat{\Sigma}^{\prime}(0)$ on the Matsubara axis. It is similar to, but not identical with the form in reference 55 on the real frequency axis, which replaces $\langle i|\hat{\Sigma}(\omega)| j\rangle$ with $\operatorname{Re}\left(\left\langle i\left|\hat{\Sigma}\left(\varepsilon_{i}\right)\right| j\right\rangle+\left\langle i\left|\hat{\Sigma}\left(\varepsilon_{j}\right)\right| j\right\rangle\right) / 2$, derived from a norm minimization principle. Here, $\varepsilon_{i}$ and $|i\rangle$ are QP energy and wavefunction, respectively.). Working on the Matsubara axis is numerically very stable, provide a natural interface with advanced DMFT solvers such as continuous-time quantum MonteCarlo, ${ }^{16,17}$ and has very good scaling in system size as in the space-time method (see Supplementary Note on MQSGW calculations).

For DMFT, it is essential to obtain bandstructures in a fine enough crystal momentum (k) mesh to attain desired frequency resolution of physical quantities. To achieve such momentum resolution, we use a Wannier-interpolated MQSGW bandstructure in a large energy window using maximally localized Wannier function ${ }^{56}$ and then constructed local projector in a fine momentum mesh. In contrast to $\mathrm{SrVO}_{3},{ }^{12-15}$ where a set of $t_{2 g}$ states is reasonably well separated from the other bands, correlated $3 d$ orbitals in $\mathrm{La}_{2} \mathrm{CuO}_{4}$ and $\mathrm{NiO}$, and are strongly hybridized with other itinerant bands. In this case, it is necessary to construct local projectors from states in a wide-enough energy windows to make projectors localized near the correlated atoms. We constructed local projectors in the energy window $E_{\mathrm{F}} \pm 10 \mathrm{eV}$ in which there are $\sim 82$ bands at each $\mathbf{k}$ point, where $E_{\mathrm{F}}$ is the Fermi level for $\mathrm{La}_{2} \mathrm{CuO}_{4}$. For $\mathrm{NiO}$, we constructed local projectors in the energy window of $E_{\mathrm{F}}-11 \mathrm{eV}$ to $E_{F}+10 \mathrm{eV}$. Next, we confirmed that absolute value of its overlap to the muffin-tin orbital (of which radial function is determined to maximize electron occupation in it) is $>95 \%$. Our choice of energy window is justified by the $\mathrm{Cu}-3 d$ spectra being entirely contained in this window. Using constructed maximally localized Wannier function $\mathrm{s}$, we defined our local projector $P_{i, n}(\mathbf{k})=\sum_{R}\left\langle W_{\mathbf{R} i} \mid \psi_{n \mathbf{k}}\right\rangle e^{-i \mathbf{k} \cdot \mathbf{R}} / \sqrt{N_{k}}$, where $W_{\mathbf{R} i}(\mathbf{r})$ is the maximally localized Wannier function with an index $i, \psi_{n \mathbf{k}}(\mathbf{r})$ is the QP wavefunction with an index $n$, and $N_{k}$ is the number of $\mathbf{k}$ points in the first Brillouin zone.

Static $U_{\mathrm{d}}$ and $J_{\mathrm{H}}$ are evaluated by a modification of the constrained random phase approximation method, ${ }^{57}$ which avoids screening by the strongly hybridized bands. This screening by hybridization is included in our large-energy window DMFT. For details, see Supplementary Note on $U_{\mathrm{d}}$ and $J_{\mathrm{H}}$. We divide dynamic polarizability within MQSGW approximation $X_{\mathrm{QP}}$ into two parts, $X_{Q P}=x_{Q P}^{\text {low }}+x_{Q P}^{\text {high }}$. Here, $X_{Q P}^{\text {low }}$ is defined by all transitions between the states in the energy window accounted for by the DMFT method $\left(E_{\mathrm{F}} \pm 10 \mathrm{eV}\right.$ for $\mathrm{La}_{2} \mathrm{CuO}_{4}$ and $E_{\mathrm{F}}-11 \mathrm{eV}$ to $E_{\mathrm{F}}+10 \mathrm{eV}$ for $\left.\mathrm{NiO}\right)$. 
Using $X_{Q P}^{\text {high }}$, we evaluate partially screened Coulomb interaction $U^{-1}\left(\mathbf{r}, \mathbf{r}^{\prime}, \mathbf{k}, i \omega_{n}\right)=V^{-1}\left(\mathbf{r}, \mathbf{r}^{\prime}, \mathbf{k}\right)-\chi_{Q P}^{\text {high }}\left(\mathbf{r}, \mathbf{r}^{\prime}, \mathbf{k}, i \omega_{n}\right)$ and parametrize static $U_{\mathrm{d}}$ and $J_{\mathrm{H}}$ by Slater's integrals, ${ }^{58,59}$ where $V$ is bare Coulomb interaction.

The Feynman graphs included in both MQSGW and DMFT (double counting) are the local Hartree and the local GW diagram. They are computed using the local projection of the MQSGW Green's function $\left(\hat{G}_{\mathrm{QP}}\right)$ $\hat{G}_{Q P}^{\text {loc }}\left(i \omega_{n}\right)=\frac{1}{N_{k}} \sum_{\mathbf{k}} \hat{P}(\mathbf{k}) \hat{G}_{Q P}\left(\mathbf{k}, i \omega_{n}\right) \hat{P}^{\dagger}(\mathbf{k})$ and the local Coulomb matrix constructed from Slater's integrals. For the details, see Supplementary Note on double counting energy.

\section{ACKNOWLEDGEMENTS}

This work was supported by the U.S Department of energy, Office of Science, Basic Energy Sicences as a part of the Computational Materials Science Program and by the Simons Foundation under the Many Electron Problem collaboration. An award of computer time was provided by the INCITE program. This research used resources of the Oak Ridge Leadership Computing Facility, which is a DOE Office of Science User Facility supported under Contract DE-AC05-00OR22725.

\section{CONTRIBUTIONS}

G.K. designed the framework of the code. S.C. developed the code, building on earlier developments of A.K and K. H. and performed the calculations. G.K., K.H. and S.C. analyzed the data with the help of A.K. and M.v.S. All authors provided comments on the paper.

\section{COMPETING INTERESTS}

The authors declare no conflict of interest.

\section{REFERENCES}

1. Anisimov, V. I. Strong Coulomb Correlations in Electronic Structure Calculations (CRC Press, 2000).

2. Georges, A., Kotliar, G., Krauth, W. \& Rozenberg, M. J. Dynamical mean-field theory of strongly correlated fermion systems and the limit of infinite dimensions. Rev. Mod. Phys. 68, 13-125 (1996).

3. Anisimov, V. I., Poteryaev, A. I., Korotin, M. A., Anokhin, A. O. \& Kotliar, G. First-principles calculations of the electronic structure and spectra of strongly correlated systems: dynamical mean-field theory. J. Phys. Condens. Matter 9, 7359 (1997).

4. Lichtenstein, A. I. \& Katsnelson, M. I. Ab initio calculations of quasiparticle band structure in correlated systems: LDA++ approach. Phys. Rev. B 57, 6884-6895 (1998).

5. Kotliar, G. et al. Electronic structure calculations with dynamical mean-field theory. Rev. Mod. Phys. 78, 865-951 (2006).

6. Almbladh, C.-O., Barth, U. V. \& Leeuwen, R. V. Variational total energies from-and-derivable theories. Int. J. Mod. Phys. B 13, 535-541 (1999).

7. Chitra, R. \& Kotliar, G. Effective-action approach to strongly correlated fermion systems. Phys. Rev. B 63, 115110 (2001).

8. Hedin, L. New method for calculating the one-particle Green's function with application to the electron-gas problem. Phys. Rev. 139, A796-A823 (1965).

9. Sun, P. \& Kotliar, G. Extended dynamical mean-field theory and GW method. Phys. Rev. B 66, 085120 (2002).

10. Biermann, S., Aryasetiawan, F. \& Georges, A. First-principles approach to the electronic structure of strongly correlated systems: combining the GW approximation and dynamical mean-field theory. Phys. Rev. Lett. 90, 086402 (2003).

11. Kotliar, G. \& Savrasov, S. Y. in New Theoretical Approaches to Strongly Correlated Systems, No. 23 in NATO Science Series (ed. Tsvelik, A. M.) 259-301 (Springer, 2001).

12. Tomczak, J. M., Casula, M., Miyake, T., Aryasetiawan, F. \& Biermann, S. Combined GW and dynamical mean-field theory: dynamical screening effects in transition metal oxides. EPL 100, 67001 (2012).

13. Sakuma, R., Werner, P. \& Aryasetiawan, F. Electronic structure of $\mathrm{SrVO}_{3}$ within GW+DMFT. Phys. Rev. B 88, 235110 (2013).

14. Taranto, C. et al. Comparing quasiparticle GW+DMFT and LDA+DMFT for the test bed material $\mathrm{SrVO}_{3}$. Phys. Rev. B 88, 165119 (2013).

15. Tomczak, J. M., Casula, M., Miyake, T. \& Biermann, S. Asymmetry in band widening and quasiparticle lifetimes in $\mathrm{SrVO}_{3}$ : competition between screened exchange and local correlations from combined gw and dynamical mean-field theory GW+DMFT. Phys. Rev. B 90, 165138 (2014).
16. Werner, P., Comanac, A., de Medici, L., Troyer, M. \& Millis, A. J. Continuous-time solver for quantum impurity models. Phys. Rev. Lett. 97, 076405 (2006).

17. Haule, K. Quantum Monte Carlo impurity solver for cluster dynamical mean-field theory and electronic structure calculations with adjustable cluster base. Phys. Rev. B 75, 155113 (2007).

18. Casula, M., Rubtsov, A. \& Biermann, S. Dynamical screening effects in correlated materials: plasmon satellites and spectral weight transfers from a Green's function ansatz to extended dynamical mean field theory. Phys. Rev. B $\mathbf{8 5}$ 035115 (2012)

19. Sun, P. \& Kotliar, G. Many-body approximation scheme beyond GW. Phys. Rev. Lett. 92, 196402 (2004).

20. Hansmann, P., Ayral, T., Vaugier, L., Werner, P. \& Biermann, S. Long-range coulomb interactions in surface systems: a first-principles description within self-consistently combined GW and dynamical mean-field theory. Phys. Rev. Lett. 110, 166401 (2013)

21. Kutepov, A., Savrasov, S. Y. \& Kotliar, G. Ground-state properties of simple elements from GW calculations. Phys. Rev. B 80, 041103 (2009).

22. Stan, A., Dahlen, N. E. \& Leeuwen, R. V. Fully self-consistent GW calculations for atoms and molecules. EPL 76, 298 (2006).

23. Stan, A., Dahlen, N. E. \& Leeuwen, R. V. Levels of self-consistency in the GW approximation. J. Chem. Phys. 130, 114105 (2009).

24. Holm, B. \& von Barth, U. Fully self-consistent gw self-energy of the electron gas. Phys. Rev. B 57, 2108-2117 (1998).

25. Hybertsen, M. S. \& Louie, S. G. Electron correlation in semiconductors and insulators: band gaps and quasiparticle energies. Phys. Rev. B 34, 5390-5413 (1986).

26. Rinke, P., Qteish, A., Neugebauer, J., Freysoldt, C. \& Scheffler, M. Combining GW calculations with exact-exchange density-functional theory: an analysis of valence-band photoemission for compound semiconductors. New J. Phys. 7 126 (2005).

27. Kotani, T., van Schilfgaarde, M. \& Faleev, S. V. Quasiparticle self-consistent GW method: a basis for the independent-particle approximation. Phys. Rev. B 76, 165106 (2007).

28. Tomczak, J. M., van Schilfgaarde, M. \& Kotliar, G. Many-body effects in iron pnictides and chalcogenides: nonlocal versus dynamic origin of effective masses. Phys. Rev. Lett. 109, 237010 (2012).

29. Tomczak, J. M. QSGW+DMFT: an electronic structure scheme for iron pnictides and beyond. J. Phys.: Conf. Ser. 592, 012055 (2015).

30. Yin, Z. P., Haule, K. \& Kotliar, G. Kinetic frustration and the nature of the magnetic and paramagnetic states in iron pnictides and iron chalcogenides. Nat. Mater. 10, 932-935 (2011).

31. Haule, K., Birol, T. \& Kotliar, G. Covalency in transition-metal oxides within all-electron dynamical mean-field theory. Phys. Rev. B 90, 075136 (2014).

32. Jarrell, M. \& Gubernatis, J. E. Bayesian inference and the analytic continuation of imaginary-time quantum Monte Carlo data. Phys. Rep. 269, 133-195 (1996).

33. Rado, G. \& Suhl, H. Magnetism: Exchange Interactions Among Itinerant Electrons, by C. Herring, Magnetism (Academic Press, 1966).

34. Schüler, M., Rösner, M., Wehling, T. O., Lichtenstein, A. I. \& Katsnelson, M. I. Optimal Hubbard models for materials with nonlocal coulomb interactions: graphene, silicene, and benzene. Phys. Rev. Lett. 111, 036601 (2013).

35. Ghijsen, J., Tjeng, L. H., Eskes, H., Sawatzky, G. A. \& Johnson, R. L. Resonant photoemission study of the electronic structure of $\mathrm{CuO}$ and $\mathrm{Cu}_{2} \mathrm{O}$. Phys. Rev. B 42 2268-2274 (1990).

36. Haule, K., Yee, C.-H. \& Kim, K. Dynamical mean-field theory within the full-potential methods: electronic structure of Celrln ${ }_{5}, \mathrm{CeColn}{ }_{5}$, and CeRhIn ${ }_{5}$. Phys. Rev. B 81, 195107 (2010).

37. Borsa, F. et al. Staggered magnetization in $\mathrm{La}_{2-x} \mathrm{Sr}_{x} \mathrm{CuO}_{4}$ from ${ }^{139} \mathrm{La}$ nqr and $\mu$ sr: effects of sr doping in the range $0<x<0.02$. Phys. Rev. B 52, 7334-7345 (1995).

38. Reehuis, M. et al. Crystal structure and high-field magnetism of $\mathrm{La}_{2} \mathrm{CuO}_{4}$. Phys. Rev. B 73, 144513 (2006).

39. Vaknin, D. et al. Antiferromagnetism in $\mathrm{La}_{2} \mathrm{CuO}_{4-y}$. Phys. Rev. Lett. 58 2802-2805 (1987).

40. Ginder, J. M. et al. Photoexcitations in $\mathrm{La}_{2} \mathrm{CuO}_{4}:$ 2-eV energy gap and long-lived defect states. Phys. Rev. B 37, 7506-7509 (1988)

41. Cooper, S. L. et al. Optical studies of gap, exchange, and hopping energies in the insulating cuprates. Phys. Rev. B 42, 10785-10788 (1990).

42. Weber, C., Haule, K. \& Kotliar, G. Optical weights and waterfalls in doped charge-transfer insulators: a local density approximation and dynamical mean-field theory study of $\mathrm{La}_{2-x} \mathrm{Sr}_{x} \mathrm{CuO}_{4}$. Phys. Rev. B 78, 134519 (2008).

43. Weber, C., Haule, K. \& Kotliar, G. Strength of correlations in electron- and hole-doped cuprates. Nat. Phys. 6, 574-578 (2010).

44. Wang, X. et al. Covalency, double-counting, and the metal-insulator phase diagram in transition metal oxides. Phys. Rev. B 86, 195136 (2012). 
45. Werner, P., Sakuma, R., Nilsson, F. \& Aryasetiawan, F. Dynamical screening in $\mathrm{La}_{2} \mathrm{CuO}_{4}$. Phys. Rev. B 91, 125142 (2015).

46. Nücker, N. et al. Experimental electronic structure studies of $\mathrm{La}_{2 x} \mathrm{Sr}_{x} \mathrm{CuO}_{4}$. Z. Phys. B Condens. Matter 67, 9-14 (1987).

47. Anisimov, V. I., Zaanen, J. \& Andersen, O. K. Band theory and mott insulators: Hubbard U instead of stoner I. Phys. Rev. B 44, 943-954 (1991).

48. Czyzyk, M. T. \& Sawatzky, G. A. Local-density functional and on-site correlations: the electronic structure of $\mathrm{La}_{2} \mathrm{CuO}_{4}$ and $\mathrm{LaCuO}_{3}$. Phys. Rev. B 49, 14211-14228 (1994).

49. Zimmermann, R. et al. Electronic structure of 3d-transition-metal oxides: on-site Coulomb repulsion versus covalency. J. Phys. Condens. Matter 11, 1657 (1999).

50. Ren, X. et al. LDA+DMFT computation of the electronic spectrum of NiO. Phys. Rev. B 74, 195114 (2006).

51. Yin, Q., Gordienko, A., Wan, X. \& Savrasov, S. Y. Calculated momentum dependence of Zhang-rice states in transition metal oxides. Phys. Rev. Lett. 100, 066406 (2008).

52. Cheetham, A. K. \& Hope, D. A. O. Magnetic ordering and exchange effects in the antiferromagnetic solid solutions $\mathrm{Mn}_{x} \mathrm{Ni}_{1-x} \mathrm{O}$. Phys. Rev. B 27, 6964-6967 (1983).

53. Fender, B. E. F., Jacobson, A. J. \& Wedgwood, F. A. Covalency parameters in MnO, MnS, and NiO. J. Chem. Phys. 48, 990-994 (1968).

54. Kutepov, A., Haule, K., Savrasov, S. Y. \& Kotliar, G. Electronic structure of Pu and Am metals by self-consistent relativistic GW method. Phys. Rev. B 85, 155129 (2012).

55. vanSchilfgaarde, M., Kotani, T. \& Faleev, S. Quasiparticle self-consistent GW theory. Phys. Rev. Lett. 96, 226402 (2006).
56. Marzari, N., Mostofi, A. A., Yates, J. R., Souza, I. \& Vanderbilt, D. Maximally localized Wannier functions: theory and applications. Rev. Mod. Phys. 84, 1419-1475 (2012)

57. Aryasetiawan, F. et al. Frequency-dependent local interactions and low-energy effective models from electronic structure calculations. Phys. Rev. B 70, 195104 (2004)

58. van der Marel, D. \& Sawatzky, G. A. Electron-electron interaction and localization in $d$ and $f$ transition metals. Phys. Rev. B 37, 10674-10684 (1988).

59. Kutepov, A., Haule, K., Savrasov, S. Y. \& Kotliar, G. Self-consistent GW determination of the interaction strength: application to the iron arsenide superconductors. Phys. Rev. B 82, 045105 (2010).

\section{(c) (i)}

This work is licensed under a Creative Commons Attribution 4.0 International License. The images or other third party material in this article are included in the article's Creative Commons license, unless indicated otherwise in the credit line; if the material is not included under the Creative Commons license, users will need to obtain permission from the license holder to reproduce the material. To view a copy of this license, visit http://creativecommons.org/licenses/ by/4.0/

(c) The Author(s) 2016

Supplemental Information accompanies the paper on the npj Quantum Materials website (http://www.nature.com/npjquantmats) 